\title{
Strategic Mating With Common Preferences
}

\author{
Steve Alpern* and Diane Reyniers** \\ *Department of Mathematics \\ ** Interdisciplinary Institute of Management \\ The London School of Economics \\ Houghton Street \\ London WC2A 2AE \\ United Kingdom
}

April 27, 2004 


\begin{abstract}
We present a two-sided search model in which individuals from two groups (males and females, employers and workers) would like to form a long term relationship with a highly ranked individual of the other group, but are limited to individuals who they randomly encounter and to those who also accept them.

This article completes the research program, begun in Alpern and Reyniers (1999), of providing a game theoretic analysis for the Kalick-Hamilton (1986) mating model in which a cohort of males and females of various 'fitness' or 'attractiveness' levels are randomly paired in successive periods and mate if they accept each other. Their model compared two acceptance rules chosen to represent homotypic (similarity) preferences and common (or 'type') preferences. Our earlier paper modeled the first kind by assuming that if a level $x$ male mates with a level $y$ female, both get utility $-|x-y|$, whereas this paper models the second kind by giving the male utility $y$ and the female utility $x$.

Our model can also be seen as a continuous generalization of the discrete fitness-level game of Johnstone (1997). We establish the existence of equilibium strategy pairs, give examples of multiple equilibria, and conditions guaranteeing uniqueness. In all equilibria individuals become less choosy over time, with high fitness individuals pairing off with each other first, leaving the rest to pair off later. This route to assortative mating was suggested by Parker (1983). If the initial fitness distributions have atoms, then mixed strategy equilibria may also occur. If these distributions are unknown, there are equilibria in which only individuals in the same fitness band are mated, as in the steady state model of MacNamara and Collins (1990) for the job search problem.
\end{abstract}




\section{Introduction}

In this paper we present a model of two-sided search in which individuals from two distinct groups seek to form a long term relationship with a member of the other group. Individuals in each group have a common preference with respect to those in the other group and seek to obtain a partner of high rank by optimal sequential rejection and acceptance of those indivuals who are randomly encountered. The two main areas of application of this model are job search (between workers and employers) and (animal or human) mate selection. We present the model and our findings in terms of the latter, and relate our work to the sociological and biological literature on mate selection with mutual choice. Readers interested in the recent economics literature in this area should look at the work of Bloch and Ryder (2000), Burdett and Coles (1997, 1999), Eeckhout (2000), McNamara and Collins (1990), and Shimer and Smith (2000).

The empirical background which provides the starting point of this article is the widespread observation, in a variety of human, animal, and economic contexts, of (positive) assortative mating. This term describes a positive correlation of a measurable trait (or traits) within various types of mated couples. The social psychologists Kalick and Hamilton (1986) developed a simulation model which produced assortative mating of a finite cohort of males and females with discrete computer generated attractiveness levels. In each of a succession of periods, unmated males and females were randomly paired, and pairs formed a mated couple if each accepted the other. They posited two types of acceptance rules: (i) accept someone with a level close to your own, and (ii) accept someone with a high level. Both types of rules produced positive correlations in inter-couple attractiveness levels roughly approximating empirical observations. This article is the second part of the authors' program of analyzing the KalickHamilton model without exogenous acceptance rules, but rather assuming only individual preferences (utilities) and obtaining corresponding acceptance rules endogenously as equilibria of the resulting dynamic game. In other words, we replace the mate selection model by a mate preference model, as distinguished in the article of Zohar and Guttman (1989). In particular, for male $(x)$ and female $(y)$ levels continuously distributed over the interval $[0,1]$ the acceptance rule (i) was replaced in our original article (Alpern \& Reyniers, 1999) by the assumption that both members of a mated pair $(x, y)$ obtained the common utility $-|x-y|$ (cost of a mating is difference in attractiveness levels). The present article completes this program by replacing acceptance rule (ii) by the assumption that each member of a mated pair receives the other's level as utility (male gets $y$, female gets $x$ ). The utilities in (i) represent what is called homotypic (or similarity) preferences, and correspond to what is known in the social psychological literature as the 'matching hypothesis', while the utilities (ii) used here are known as maximizing (or type) preferences.

Subsequent to the bulk of our work presented here, the article of Johnstone (1997) has come to our attention. His pioneering work can be seen as an earlier game theoretic discrete version of the maximizing-preference case of the KalickHamilton model, and in this context the present article is a continuous version 
of the Johnstone model. However there is no overlap in results, as our article is mainly analytical whereas Johnstone's work is mainly computational. In this perspective our formal results give a theoretical underpinning for the conclusions given by Johnstone for a wide variety of biologically significant questions.

The aim of this article is to complete the program of game theoretic analysis of the Kalick-Hamilton model by analyzing the maximizing preference case of their model in terms of a dynamic game $\Gamma_{n}\left(F_{1}, G_{1}\right)$. In this game initial populations of males and females (the cohort) have their fitness levels distributed of the interval $[0,1]$ according to respective cumulative probability distributions $F_{1}$ and $G_{1}$. We give special attention to the symmetric case of identical initial distributions, which we denote by $\Gamma_{n}\left(F_{1}\right)=\Gamma_{n}\left(F_{1}, F_{1}\right)$ and to the special symmetric case denoted simply $\Gamma_{n}$ where the common distribution is uniform. In each of $n$ rounds, unmated males and females are randomly paired, and become mated if there is mutual acceptance. A strategy for say a male of level $x$ says which females to accept (if paired with) in each period $k=1, \ldots, n$. A strategy profile, giving such rules for all males and females, is said to be an equilibrium if say a male $x$ accepts in period $k$ only those females whose level exceeds the expected level of a female he will be mated with if he plays optimally in subsequent rounds. We establish the existence of such equilibria for all continuous initial distributions $F_{1}$ and $G_{1}$, and give examples to demonstrate that there may be more than one equilibrium. We show that in any equilibrium, each period $k$ is associated with a pair of acceptance points $a_{k}$ and $b_{k}$ (both decreasing in $k$ ) with the property that the only couples formed in period $k$ consist of females above $a_{k}$ and males above $b_{k}$. This mating pattern differs from that found in the similar steady state model (not a fixed cohort but with equal numbers entering and coupling in each period) of MacNamara and Collins (1990), who found that couples formed if and only if the paired male and female belongs to the same band (interval) of levels, where these interval bands partitioned the full spectrum of possible levels. Within these bands, mating was random. Our previous paper on homotypic preferences found that at equilibrium individual threshold acceptance levels (in that case, how far from one's one value to accept) varied continuously with 'attractiveness', as opposed to the banding we find here. Johnstone (1997) asserts that his model also predicts continuous changes in threshold levels. Since his model is very similar to the one presented here we assume that this difference arises from his use of probabilistic acceptance rules.

The equilibrium mating pattern found here corresponds to that posited by Parker (1983), in that individuals with high (fitness) levels pair off with each other first, leaving the lower fitness individuals to pair off with each other later. (A similar observation was made by Johnstone (1997) regarding his model.) This results in a gradual lowering over time of the fitness levels in the unmated pool. We prove that the acceptance levels of both males and females are decreasing over time, so that they get 'less choosy'. This is of course equivalent to a point of view in which acceptance levels remain constant, but that perceptually observed fitness levels are increasing over time. This has been empirically observed in the country and western song "Don't the girls get prettier at closing time" as studied by Pennebaker et. al. (1979), and in terms of animal breeding seasons 
by Real (1990).

An important theoretical and practical question relates to symmetry, or the lack of it, between males and females. Our model allows for different initial distributions of fitness among males and females $\left(F_{1} \neq G_{1}\right)$, and we establish our general results in this context. However to reduce the complexity of the analytical solutions, our worked out examples mainly consider the symmetric case. Even here it is not clear a priori that this ensures that equilibria must be symmetric - it is theoretically possible that asymmetric equilibria exist in such cases, though we have not found any. In the other direction, we have established limited results that symmetric problems have only symmetric equilibria, for example when $n=2$ or when $n=3$ and the common initial distribution is uniform. Johnstone (1996) explores this problem in great detail, especially in the biologically significant area of sex differences in parental care.

We find that if the initial distributions $F_{1}, G_{1}$ of fitness are not known but can be gradually learned, considering the pairings as a sampling process, then we do obtain a coupling pattern similar to that found by MacNamara and Collins (1990) in their steady state infinite horizon model. In that pattern males and females are segmented into quality bands and mate only if they are in corresponding (say both top) bands. We consider also the possibility that individuals do not know their own fitness levels, but learn this over time through observations about which individuals accept them. However the type of equilibrium which we find always prevails can be implemented even by individuals who do not know their own type, so our model does not seem to allow this type of self-learning to be studied.

We also show how mixed strategy equilibria, where an individual's strategy may require accepting a potential mate of some level with a non-trivial probability, may exist. In our model these mixed equilibria occur endogenously in the model, as opposed to the versions of Kalick \& Hamilton (1986) and Johnstone (1997) where probabilistic acceptance strategies are exogenously incorporated into the model. However mixed strategies can only exist when one of the initial distributions $F_{1}$ or $G_{1}$ has an atom (a positive probability of some fitness level).

Our analysis also deals with asymmetric problems. For example, we show that when the females only are given a charge $c$ (negative utility) to go into period 2 in $\Gamma_{2}$, not only the females become less choosy in the first period (obvious), but also the males. Much more analysis of asymmetric problems can be found, in an alternative model, in Johnstone et al (1996).

Johnstone (1997) observes that when both sexes exercise choice, a game theoretic model must be adopted (also citing Parker, 1983, and Crowley et al, 1991, to this effect), because "the best strategy for males depends on the behavior of females, and vice versa". In fact when heterogenous fitness levels are assumed for both sexes, the game is not simply one between males and females, and an individual's best behavior also depends on the behavior of other individuals of his/her own sex but with different fitness levels. Thus the game presented here should be see as one having a continuum of players, distributed between two sexes. Conflicts of interest between the sexes have also been observed in the context of alternative stable matchings by Bergstrom and Real (2000), where 
an extensive theory of mutual mate choice is presented.

\section{The Game $\Gamma_{n}\left(F_{1}, G_{1}\right)$}

In this section we formally define the general cohort mating game $\Gamma_{n}\left(F_{1}, G_{1}\right)$ and its two special cases; the symmetric game $\Gamma_{n}\left(F_{1}\right)=\Gamma_{n}\left(F_{1}, F_{1}\right)$ and the symmetric uniform distribution game $\Gamma_{n}=\Gamma_{n}$ (uniform). The game $\Gamma_{n}\left(F_{1}, G_{1}\right)$ is essentially a continuous generalization of the Kalick-Hamilton-Johnstone discrete mating models. There are $n$ periods. We assume that initially (at the beginning of period 1) males have their fitness (replacing Kalick's term 'attractiveness') $x$ distributed on $[0,1]$ according to a continuous cumulative probability distribution $F_{1}$, and similarly female fitness $y$ is initially distributed according to a similar distribution $G_{1}$. Examples with atoms (positive probability of a particular fitness level) will sometimes be considered, because they are easier to analyze. We assume that these distributions are known, though Section 7 explores the role of learning in the alternative case. For simplicity, we assume that the total populations of males and females are equal in each round (though unequal populations can also be analyzed in this model by making the reservation values described below positive). In each of $n$ rounds, unmated males and females are randomly paired - this is called a matching. Saying that the matching is random simply means that the probability of any male $x$ being matched with a female in fitness interval $Y$ in round $m$ is simply the fraction of females with fitness levels in $Y$ at the beginning of that round. If matched individuals accept each other, then they leave the pool and each receive the other's fitness as their own payoff. This is called a mating. We could incorporate exogenous costs of waiting (going into the next period), in order to encourage acceptance. For example, we could make the utility of a mating $(x, y)$ in period $m$ depend explicitly on $m$. For example the male's utility could be $y-c(m-1)$ if there is a fixed cost $c$ of entering each period, or $d^{m-1} y$ if there is a discounting of the female's value over time ( $c=0$ and $d=1$ reduce to the original case). We examine the effect of such costs on the inter-couple correlation coefficient (which can go either way). We note that if the fixed cost $c$ is sufficiently large, then the 'universal acceptance' strategy will dominate. A male who leaves the final round unmated receives utility $z_{n+1}$ (similarly a female receives $w_{n+1}$ ) We will usually take these reservation values to be zero, for simplicity. In this case there will obviously be mutual acceptance in the final ( $n$ 'th) round, so that the game will end with all individuals mated.

Note that there is some flexibility in using initial distributions $\left(F_{1}, G_{1}\right)$ or final utilities ( the reservation values $w_{n+1}$ and $z_{n+1}$ ) to describe the same situation. For example, suppose that the initial distributions of males and females are both uniform on $[0,1]$ and that the utility for any individual of leaving the final round unmated is given by $w_{n+1}=z_{n+1}=1 / 4$. Then no individual with fitness below $1 / 4$ will ever be accepted, so their fitness could just as well be given as 0 (or negative). In this altered model, we would have the initial distributions with an atom of probability $1 / 4$ at zero, and the rest 
uniformly distributed on $[1 / 4,1]$, with the value of leaving the final period unmated as 0 . So it would be sufficient to analyze a model in which we are given that $w_{n+1}=z_{n+1}=0$, but for ease of applicability we considered the more general case here. The same type of analysis allows us to deal with unequal initial (and hence subsequent) populations of males and females. If there are more males than females, we could add 'phantom' females of zero (or negative) fitness. Being matched with one of these phantoms would be equivalent to being unmatched in that period. Our model can have everyone matched in each round for this reason.

We now consider what a strategy might look like for an individual playing this game. A strategy pair $(f, g)=\left(\left(f_{1 \ldots \ldots} f_{n-1}\right),\left(g_{1}, \ldots, g_{n-1}\right)\right)$ is defined as follows. If a male of level $x$ (simply called a male $x$ or even just $x$ ) meets a female $y$ in period $m, x$ will accept $y$ iff $y \geq f_{m}(x)$ and $y$ will accept $x$ iff $x \geq g_{m}(y)$. Hence $x$ and $y$ will be mated (and leave the pool) iff both conditions are satisfied. More general strategies could have been considered, such as letting $f_{m}(x)$ be an arbitrary subset of $[0,1]$, but the definition we choose is simple and sufficiently general that it will not exclude any equilibrium pairs of more general strategies.

Given a strategy pair $(f, g)$ one can easily calculate the normalized population distributions $F_{m}$ and $G_{m}$ of males and females at the beginning of each period $m$. This enables us to calculate the expected value $v_{m}^{1}(x)$ for a male of fitness $x$ who is unmated at the start of period $m$ - this gives the expected fitness of the female he is eventually mated with. Similarly there is a female value function $v_{m}^{2}(y)$. Clearly both $v^{1}$ and $v^{2}$ depend on the strategy pair $f, g$.

A pair $(f, g)$ is called an equilibrium if for all $m=1,2 \ldots, n-1$ and all $x$ and $y$, we have

$$
\begin{aligned}
& f_{m}(x)=v_{m+1}^{1}(x), \text { and } \\
& g_{m}(y)=v_{m+1}^{2}(y) .
\end{aligned}
$$

The first equation simply means that at equilibrium a male of type $x$ will accept a female of type $y$ in period $m$ if and only if $y$ exceeds the expected fitness of the mate he will obtain if he goes into the next $(m+1$ 'th) period unmated. This definition of equilibrium is somewhat analogous to a subgame perfect equilibrium of a finite extensive form game. We observe that in the final period $n$ any match will be accepted if the reservation values are zeros, or more generally, any match above the reservation value will be accepted. That is, the equilibrium condition for the final period $n$ is defined by setting $v_{n+1}^{1}=z_{n+1}$ and $v_{n+1}^{2}=w_{n+1}$.

There are some caveats to this definition that will apply to pathological (from our perspective) initial distributions. As long as the probability of meeting any given type is zero ( $F_{1}$ and $G_{1}$ are non-atomic distributions) we do not need to worry about the indifference case where $x$ meets a $y$ satisfying $y=v_{m+1}^{1}(x)$. However if there is an atom at $y$, we will need to consider mixed strategies where $x$ accepts such a $y$ with a non-trivial probability. We postpone the discussion of such strategies until Section 6. Another observation about the equilibrium condition (1) is that if say there are no females with fitness in the interval $(.3, .6)$, 
(that is, if $\left.G_{1}(.3)=G_{1}(.6)\right)$ then the strategy with $f_{m}(x)=.4$ and the one with $f_{m}(x)=.5$ (for some $x$ ) are equivalent in the sense that they accept the same set of females. We will not consider equilibrium strategy pairs which differ only in this trivial sense to be examples of non-unique equilibria. (We will find true examples of non-uniqueness in Section 6).

We will sometimes consider a version of the model which is symmetric with respect to males and females in that the two initial distributions $F_{1}$ and $G_{1}$ are identical. We shall denote this symmetric game $\Gamma_{n}\left(F_{1}, F_{1}\right)$ simply by $\Gamma_{n}\left(F_{1}\right)$, so if there is only one argument this will denote symmetry. It is theoretically possible that in this case there still might exist asymmetric equilibria (i.e., with $f \neq g$ ), although we have a limited result in the other direction in Theorem 4. The symmetric game is simpler to analyze analytically, as there are only half as many variables to solve for. The symmetric game also may be applied to the situation where there is a single population which needs to be paired (e.g., people looking for good tennis partners, among randomly encountered players).

For the symmetric game we will consider mainly symmetric equilibria, those with $f=g$, and in this case we use the simpler notation $v=v^{1}=v^{2}$. So the equilibrium condition (1) becomes simply

$$
f_{m}(x)=v_{m+1}(x), m=1, \ldots, n .
$$

A special case of the symmetric game is the version where the initial distribution for males and females is uniform on $[0,1]$, that is, $F_{1}(t)=G_{1}(t)=t$. We shall denote this game by $\Gamma_{n}$. This special game is useful for two reasons. First of all, the calculations are short. But more important is the fact that this is the distribution used in Kalick's dating simulation model and also in the authors' earlier paper. Our comparisons between similarity (homotypic) and maximizing preferences are made easier if we use the same distribution.

\section{Analysis of $\Gamma_{2}, \Gamma_{3}$}

In this section we derive the equilibrium strategies for the symmetric mating games with initial uniform distributions of fitness and $n=2$ or 3 periods, $\Gamma_{2}$ and $\Gamma_{3}$. We also consider the effect of an entry cost $c$ on equilibrium acceptance levels and on inter-couple correlation. We will also consider the possibility of asymmetric equilibria.

For $n=2$ only $f_{1}$ is in doubt, and this will be given by as a constant equal to the final period mean fitness level. If individuals above the value $u$ are accepted in the first round $\left(f_{1}=u\right)$ an individual $x$ with $x<u$ will definitely stay in the unmated pool for period 2, and an individual $x$ with $x>u$ will stay in it with probability $u$. That is, he will stay unmated if and only if he is matched with someone below $u$. So the total population (of both males and females) at the beginning of period 2 will be

$$
1(u)+u(1-u)=2 u-u^{2} .
$$


Hence the mean fitness in the final (second) round will be

$$
\begin{aligned}
\left(\int_{0}^{u} x d x+u \int_{u}^{1} x d x\right) /\left(2 u-u^{2}\right) & =\frac{\frac{1}{2} u^{2}+u\left(\frac{1}{2}-\frac{1}{2} u^{2}\right)}{2 u-u^{2}} \\
& =\frac{1}{2}\left(\frac{-u-1+u^{2}}{-2+u}\right)
\end{aligned}
$$

At a symmetric equilibrium (2) this will equal the cutoff acceptance value $u$, so we set

$$
\begin{aligned}
u & =\frac{1}{2}\left(\frac{-u-1+u^{2}}{-2+u}\right), \text { or } \\
u & =\frac{3}{2}-\frac{1}{2} \sqrt{5}=.38197 .
\end{aligned}
$$

The mean fitness in period 2 as a function of the acceptance value $f_{1}=u$ in period 1 is shown in Figure 1 below, where the (unique) intersection with the 45 degree line denotes the equilibrium strategy $u$.

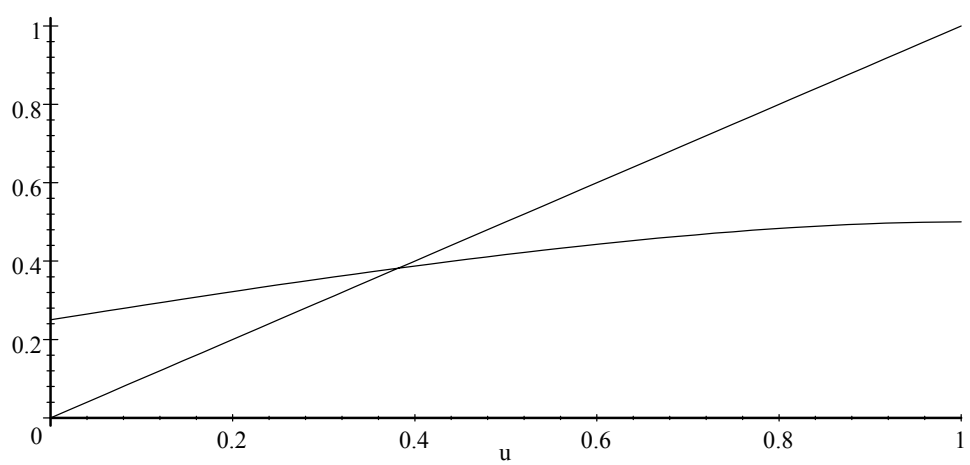

Figure 1: Mean fitness in period 2 as function of choosiness.

If the acceptance level in the first period is $u$ (for both sexes), $0<u \leq 1$, then the resulting inter-couple correlation coefficient when everyone is mated after period 2 is given by

$$
\rho(u)=\frac{u(1-u)^{2}(4 u+1)}{2-u},
$$

which peaks at $1 / 4$ when $u=1 / 2$, as shown below in Figure 2. Note in particular 
that $\rho$ is increasing up to $1 / 2$. (This fact will be used later.)

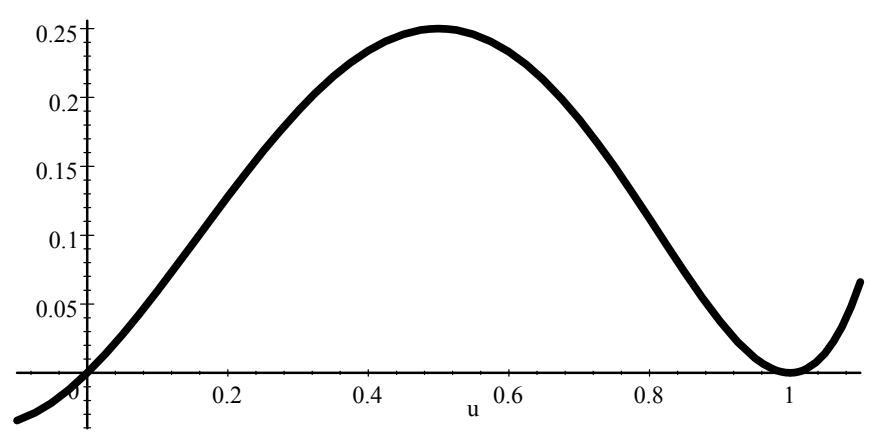

Figure 2: Inter-couple correlation in terms of choosiness

If there is a fixed cost $c$ of entering each period, or a constant factor $d$ which discounts the value of a given level mate in each period, then the equilibrium equation (3) becomes respectively

$$
u=\frac{1}{2}\left(\frac{-u-1+u^{2}}{-2+u}\right)-c \text { and } u=d \frac{1}{2}\left(\frac{-u-1+u^{2}}{-2+u}\right)
$$

and the resulting equilibrium acceptance level $u$ for the first period is as plotted in Figures 3 and 4 . Note that when $c=0$ or $d=1$ the original value of .381 obtains.

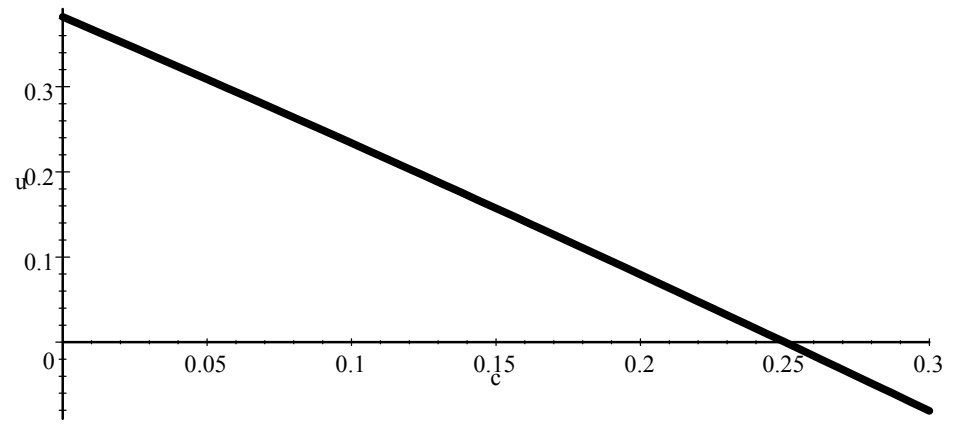

Figure 3: Equilibrium with fixed entry cost $c$. 


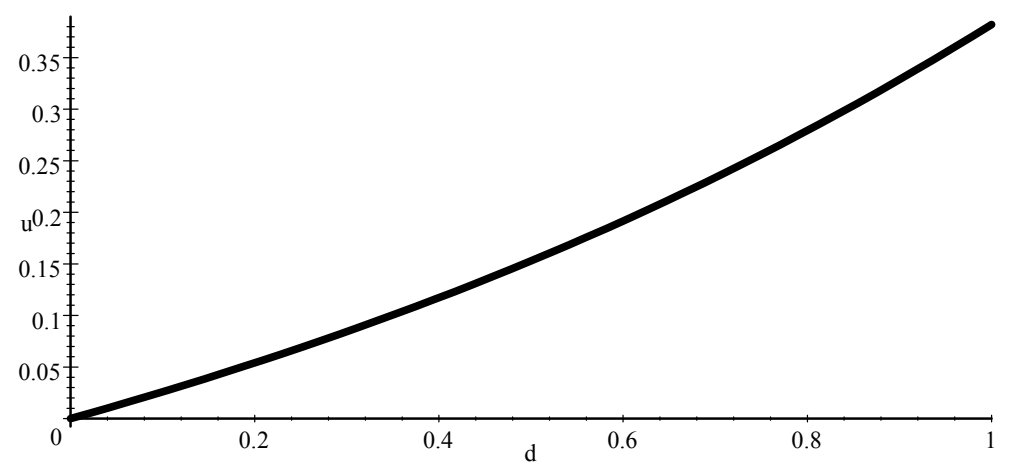

Figure 4: Equilibrium with discount factor $d$.

Note that as all the equilibrium acceptance levels are below $1 / 2$, it follows from the increasing nature of $\rho$ (see Figure 2) that the inter-couple correlation is decreasing in $c$ and increasing in $d$.

\section{1 asymmetric strategies, $\mathrm{n}=2$}

We now analyze the $n=2$ game allowing for the possibility of asymmetric equilibrium strategies. Our analysis will demonstrate that there aren't any. Consider a generic period 1 asymmetric strategy pair $(a, b)$ in which a male $x$ accepts any female of fitness level $y \geq a$ and a female $y$ accepts $x \geq b$. The pairs $(x, y)$ in the shaded rectangle of area $(1-a)(1-b)$ of Figure 5 mate in period 1.

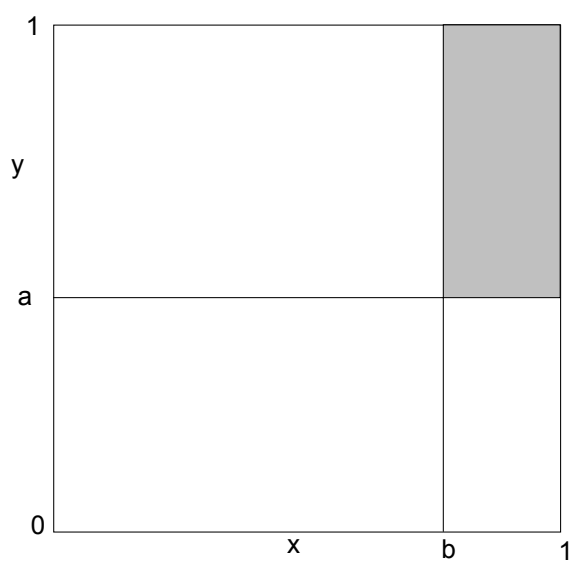

Figure 5: Asymmetric mating pattern 
Hence the total population (of each sex) at the beginning of period 2 is $1-$ $(1-a)(1-b)=b+a-a b$. The mean of the male period 2 population is calculated as follows. The 'low' males (below $b$ ) have average fitness $b / 2$, and they form an interval of length $b$ of full density 1 , the 'high' males have average fitness $(1+b) / 2$, form an interval of length $1-b$, and have density $a$ (since a fraction $1-a$ of them have been mated in period 1 ). Hence the mean male fitness level in period 2 is given by

$$
\frac{(b / 2)(b) 1+((1+b) / 2)(1-b) a}{b+a-a b}=\frac{1}{2} \frac{-b^{2}-a+a b^{2}}{-b-a+a b}
$$

Setting this mean period 2 male fitness level equal to the female period 1 acceptance level $b$ gives the female equilibrium equation (lower equation of (1))

$$
b=\frac{1}{2} \frac{-b^{2}-a+a b^{2}}{-b-a+a b} .
$$

By symmetry, the male period 1 acceptance level is given by the female period 2 mean,

$$
a=\frac{1}{2} \frac{-a^{2}-b+b a^{2}}{-a-b+b a} .
$$

These two curves are shown together with the line of symmetry $b=a$ in Figure 6 , the top equation (for $b$ ) drawn with a thicker line. (For the moment, ignore the dotted line as it is only relevant in the next paragraph.) The only intersection corresponds to the symmetric equilibrium $a=b=\frac{3}{2}-\frac{1}{2} \sqrt{5}=.38197$ found earlier. The fact that the two equilibrium curves have a single point of intersection is a special case of Theorem 4 for the uniform distribution.

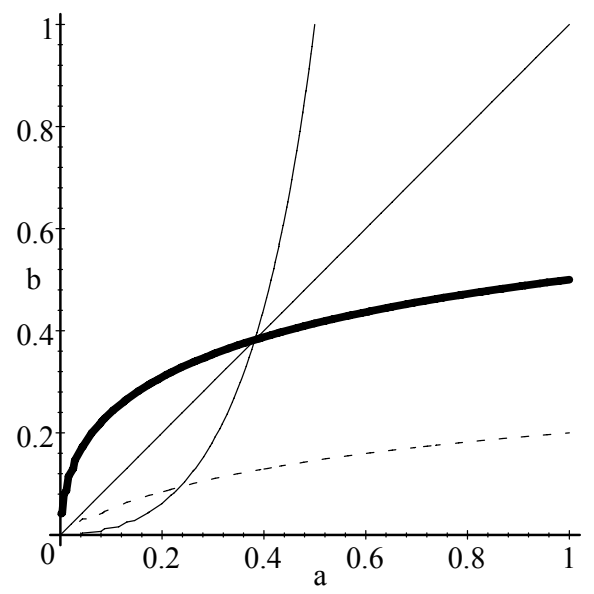

Figure 6: Male and female equilibrium equations 
If we make the problem asymmetric by imposing a per-period cost $c$ on the females, $0<c<1 / 2$, but no cost on the males, then the equilibrium is obtained by simultaneously solving the original male equilibrium equation (5) and the female equation (4) with $c$ subtracted from the right hand side. The unique solution is given by period one male and female acceptance levels $a$ and $b$, where

$$
\begin{aligned}
a & =\frac{b}{1-2 c}, \text { and } \\
b & =\frac{1}{2}(3-4 c-\sqrt{(-8 c+5)}) .
\end{aligned}
$$

In period 1 we see that females, as expected, become less choosy ( $b$ is decreasing in $c$ ). In fact for $c=1 / 2$ females accept all males. It is less obvious that males, while in all cases choosier than females $(a>b)$, also become less choosy ( $a$ is also decreasing in $c$ ). The explanation can be seen in Figure 6, now paying attention to the dotted line representing the female equilibrium equation when $c=.3$. The new intersection with the unchanged male (thin) equilibrium curve has a smaller $a$ value and a smaller $b$ value. To understand the limiting case $c=1 / 2$, where females are universal acceptors, suppose males accept females above $a$ in period 1 . Then in period 2 females are uniformly distributed on $[0, a]$, with mean $a / 2$. Hence the equilibrium equation for the males is $a=a / 2$, with solution $a=0$. Hence males are also universal acceptors. This is in accordance with the above equilibrium formula, as it has a limiting value for $a$ of 0 as $c$ approaches $1 / 2$.

\subsection{Analysis of $\Gamma(3)$}

If we denote by $w$ and $u$ the acceptance levels in periods 1 and 2 , the equilibrium equations for these periods become

$$
\begin{aligned}
w & =\frac{u^{2}+w+w^{2}-w^{3}}{4 w-2 w^{2}}, \text { and } \\
u & =\frac{u^{2}+u(-2+w) w+w\left(-1-w+w^{2}\right)}{2(u+2(w-2) w)} .
\end{aligned}
$$

There is a unique solution (equilibrium), given by

$$
\begin{aligned}
& u=\frac{1}{27}(23-\sqrt{205}) \doteq .3216 \\
& w=\frac{1}{18}(23-\sqrt{205}) \doteq .4823
\end{aligned}
$$

\section{Properties of Equilibria}

In this section we demonstrate that while in general strategies for the cohort mating game may have many forms, equilibrium strategies are always of a particular 'stepwise' type. The following result gives our characterization of equilibrium strategies. 
Theorem 1 Every equilibrium $(f, g)$ in the game $\Gamma_{n}\left(F_{1}, G_{1}\right)$ (with given reservation values $w_{n+1}$ and $\left.z_{n+1}\right)$ is characterized by two sequences

$$
\begin{aligned}
& 0=w_{n+2} \leq w_{n+1}<w_{n}<\cdots<w_{2}<w_{1}=1 \text { and } \\
& 0=z_{n+2} \leq z_{n+1}<z_{n}<\cdots<z_{2}<z_{1}=1, \text { such that }
\end{aligned}
$$

$x$ and $y$ are mutually acceptable in period $i$ iff $x>w_{i+1}$ and $y>z_{i+1} \cdot(6)$

Furthermore the strategy pair $(f, g)$ is determined by these sequences according to the following rules. In period $i=1, \ldots, n$, a male $x$ will accept a female $y$ iff $y \geq f_{i}(x)$, where.

$$
f_{i}(x)= \begin{cases}z_{k}, & \text { if } x \in\left(w_{k+1}, w_{k}\right), k \geq i+2 \\ z_{i+1}, & \text { if } x>w_{i+2},\end{cases}
$$

and a female $y$ will accept a male $x$ iff $x \geq g_{i}(y)$, where

$$
g_{i}(y)= \begin{cases}w_{k}, & \text { if } y \in\left(z_{k+1}, z_{k}\right), k \geq i+2 \\ w_{i+1}, & \text { if } y>z_{i+2} .\end{cases}
$$

Proof. First note that the claimed conditions $(7,8)$ imply the claimed mating condition (6). For example, the pattern of acceptances at equilibrium is shown in Figure 7 for period $i=3$ for the case $n=7$ and $w_{n+1}=z_{n+1}=0$. The claimed acceptance pattern $f_{3}(x)$ is shown within the figure enclosed by the thick lines, and the resulting mating set is indicated by the lightly shaded 
area.

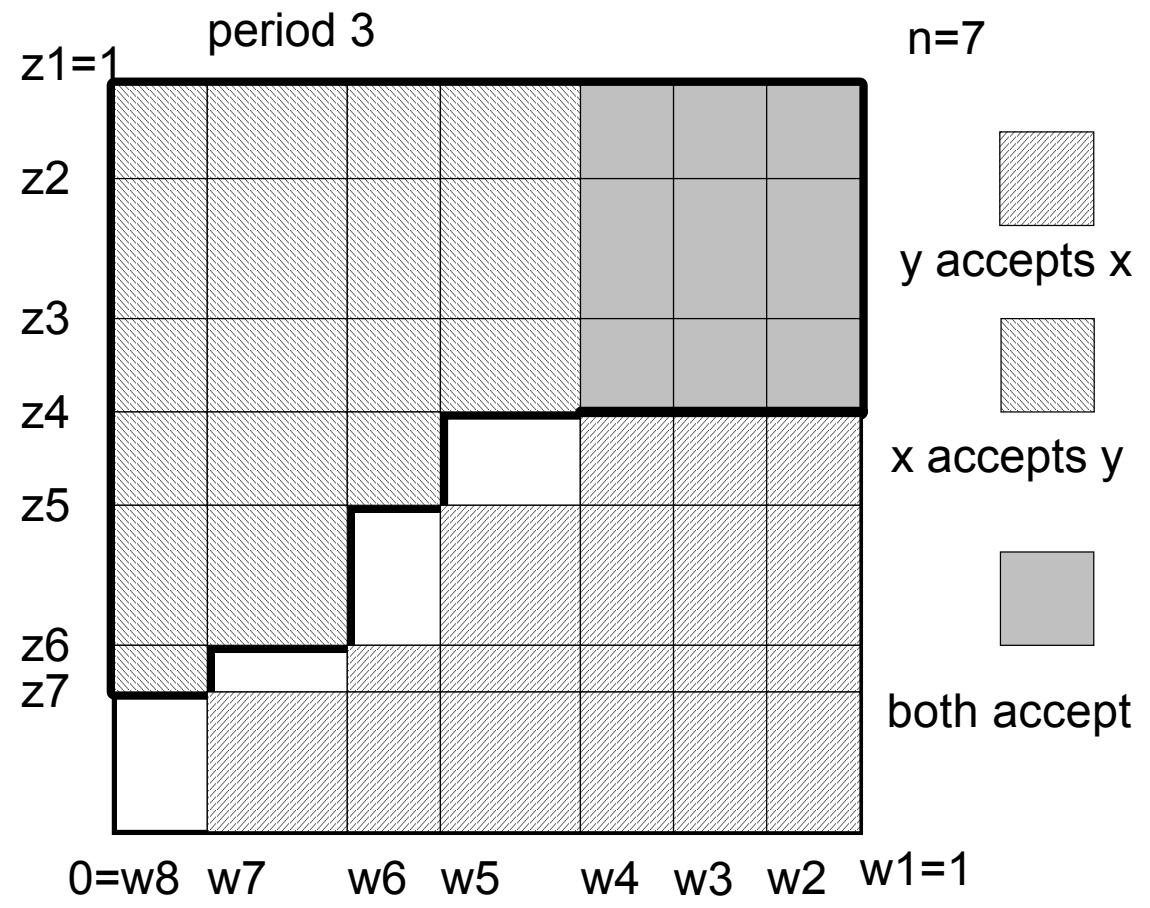

Figure 7: Equilibrium mating pattern in period 3 of 7.

So it is sufficient to establish the conditions $(7,8)$, which we do by backward induction on the period $i=n-1, n-2, \ldots, 1$.

The strategy pair $(f, g)$ and the initial distributions $F_{1}$ and $G_{1}$ together determine all the subsequent distributions $F_{i}$ and $G_{i}, i=2, \ldots, n$. Given these distributions and the given values $w_{n+1}=z_{n+1}$ (usually taken to be 0 ), we determine the remaining values $w_{n}, w_{n-1}, \ldots, w_{2}$ and $z_{n}, z_{n-1}, \ldots, z_{2}$ backwards by the recursive equations

$$
\begin{aligned}
& w_{i}=w_{i+1} F_{i}\left(w_{i+1}\right)+\int_{w_{i+1}}^{1} x d F_{i}(x), \text { and } \\
& z_{i}=z_{i+1} G_{i}\left(z_{i+1}\right)+\int_{z_{i+1}}^{1} y d G_{i}(y)
\end{aligned}
$$

First note that in the usual case that $w_{n+1}=z_{n+1}=0$ we have that

$$
w_{n}=\int_{0}^{1} x d F_{n}(x) \text { and } z_{n}=\int_{0}^{1} y d G_{n}(y)
$$

are the mean fitness levels for the final period $(i=n)$ males and females. Since all matches in the final period are mutually acceptable, the expected value for 
any male entering this period unmated will be $z_{n}$, and similarly $w_{n}$ for any such female. More generally, with given non-negative reservation values $z_{n+1}$ and $w_{n+1}$, a female $y \geq z_{n+1}$ entering the final period unmated will obtain the reserve value $w_{n+1}$ if she is matched with a male $x<w_{n+1}$ and will obtain utility $x$ if she meets a male $x \geq w_{n+1}$. So her expected value is given by

$$
v_{n}^{2}(y)=w_{n+1} F_{n}\left(w_{n+1}\right)+\int_{w_{n+1}}^{1} x d F_{n}(x)=w_{n},
$$

with a corresponding result for a male $x \geq w_{n+1}$. Note that since

$$
\begin{aligned}
w_{n+1} & =w_{n+1} F_{n}\left(w_{n+1}\right)+\int_{w_{n+1}}^{1} w_{n+1} d F_{n}(x), \text { we have from above that } \\
w_{n}-w_{n+1} & =\int_{w_{n+1}}^{1}\left(x-w_{n+1}\right) d F_{n}(x) \geq 0 \text { and positive if } F_{n}\left(w_{n+1}\right)<1 .
\end{aligned}
$$

A female $y<z_{n+1}$ will leave the period $n$ unmated and receive the exogenously given reserve value $w_{n+1}$. Hence for any $x$ and $y$ we have

$$
\begin{gathered}
v_{n}^{1}(x)=\left\{\begin{array}{ll}
z_{n+1}, & \text { if } x<w_{n+1}, \\
z_{n}, & \text { if } x \geq w_{n+1},
\end{array}\right. \text { and similarly } \\
v_{n}^{2}(y)= \begin{cases}w_{n+1}, & \text { if } y<z_{n+1}, \\
w_{n}, & \text { if } y \geq z_{n+1} .\end{cases}
\end{gathered}
$$

Consequently the equilibrium conditions (1) give

$$
\begin{aligned}
& f_{n-1}(x)= \begin{cases}z_{n+1}, & \text { if } x<w_{n+1}, \\
z_{n}, & \text { if } x \geq w_{n+1},\end{cases} \\
& g_{n-1}(y)= \begin{cases}w_{n+1}, & \text { if } y<z_{n+1}, \\
w_{n}, & \text { if } y \geq z_{n+1} .\end{cases}
\end{aligned}
$$

In period $n-1$ a pairing $(x, y)$ will mate if and only if $x \geq w_{n}$ and $y \geq z_{n}$. These conditions coincide with the claimed results for $i=n-1$.

Now suppose that the results $(7,8)$ and $w_{i+1}>w_{i+2}$ have been established for $i \geq m+1$. Under this assumption we will establish these results for $i=m$, which will prove the theorem by induction. We have to evaluate the value $v_{m+1}^{1}(x)$ of a male of type $x$ entering the $m+1$ 'st period unmated. (A corresponding evaluation for $v_{m+1}^{2}(y)$ can be similarly obtained.). According to the induction hypotheses for $i=m+1$, a male of type $x>w_{m+2}$ will be mated in period $m+1$ if any only if he is matched with a female of type $y>z_{m+2}$. Otherwise he will go into the next period $m+2$ and obtain an expected value of $v_{m+2}^{1}(x)$. Consequently if $x>w_{m+2}$ we have

$$
v_{m+1}^{1}(x)=v_{m+2}^{1}(x) G_{m+1}\left(z_{m+2}\right)+\int_{z_{m+2}}^{1} y d G_{m+1}(y) .
$$


However if $x>w_{m+2}$ we also have $x>w_{m+3}$ by the induction hypothesis, so that by the hypotheses $(7,8)$ we have $v_{m+2}^{1}(x)=f_{m+1}(x)=z_{m+2}$. So the above equation can be written simply as

$$
v_{m+1}^{1}(x)=z_{m+2} G_{m+1}\left(z_{m+2}\right)+\int_{z_{m+2}}^{1} y d G_{m+1}(y)=z_{m+1} .
$$

Consequently the equilibrium equation (1) gives

$$
f_{m}(x)=z_{m+1} \text { for } x>w_{m+2} .
$$

If $x<w_{m+2}$, then for some $k \geq m+2$, we have that $x \in\left(w_{k+1}, w_{k}\right)$. According to our inductive hypothesis (6) for period $i=m+1$, such a male $x$ will not be mated in period $m+1$. Hence we have that

$$
v_{m+1}^{1}(x)=v_{m+2}^{1}(x)=f_{m+1}(x) .
$$

But we know $f_{m+1}(x)$ from the top line of the induction hypotheses as

$$
f_{m+1}(x)=z_{k}, \quad \text { if } x \in\left(w_{k+1}, w_{k}\right), k \geq m+3 .
$$

If $x \in\left(w_{k+1}, w_{k}\right)$ for $k=m+2$, that is, if $x \in\left(w_{k+3}, w_{k+2}\right)$, then by the bottom line of the inductive hypothesis we have that

$$
\begin{aligned}
& f_{m+1}(x)=z_{m+2} \text { if } x>w_{m+3}, \text { or } \\
& f_{m+1}(x)=z_{k} \text { if if } x \in\left(w_{k+1}, w_{k}\right), k=m+2 .
\end{aligned}
$$

Hence we have from $(12,13,14)$ that

$$
v_{m+1}^{1}(x)= \begin{cases}z_{k}, & \text { if } x \in\left(w_{k+1}, w_{k}\right), k \geq m+2, \\ z_{m+1}, & \text { if } x>w_{m+2} .\end{cases}
$$

Setting

$$
f_{m}(x)=v_{m+1}^{1}(x)= \begin{cases}z_{k}, & \text { if } x \in\left(w_{k+1}, w_{k}\right), k \geq m+2, \\ z_{m+1}, & \text { if } x>w_{m+2} .\end{cases}
$$

gives the required formula for $f_{m}(x)$. The required formula for $g_{m}(x)$ can be obtained in a similar manner. It remains only to establish that $w_{m+1}>w_{m+2}$ under the inductive hypothesis that $w_{m+2}>w_{m+3}$. First recall the two specific definitions from (9):

$$
\begin{aligned}
& w_{m+1}=w_{m+2} F_{m+1}\left(w_{m+2}\right)+\int_{w_{m+2}}^{1} x d F_{m+1}(x), \text { and } \\
& w_{m+2}=w_{m+3} F_{m+2}\left(w_{m+3}\right)+\int_{w_{m+3}}^{1} x d F_{m+2}(x) .
\end{aligned}
$$

The two equations involve the three intervals $L=\left(0, w_{m+3}\right), M=\left(w_{m+3}, w_{m+2}\right)$, and $R=\left(w_{m+2}, 1\right)$ (left, middle, right), and the two distributions $F_{m+1}$ and 
$F_{m+2}$. Since $F_{m+2}$ is obtained from $F_{m+1}$ by removing some males above level $w_{m+2}$ and renormalizing, the associated measures satisfy $\mu_{m+2}(R)<\mu_{m+1}(R)$, $\mu_{m+2}(M)>\mu_{m+1}(M)$, and $\mu_{m+2}(L)>\mu_{m+1}(L)$. Let $M^{*}$ and $R^{*}$ denote the center of mass of intervals $M$ and $R$ (the same with respect to either measure). With this easier notation we may rewrite $w_{m+1}$ and $w_{m+2}$ as

$$
\begin{aligned}
& w_{m+1}=w_{m+2} \mu_{m+1}(L \cup M)+R^{*} \mu_{m+1}(R), \text { and } \\
& w_{m+2}=w_{m+3} \mu_{m+2}(L)+M^{*} \mu_{m+2}(M)+R^{*} \mu_{m+2}(R) .
\end{aligned}
$$

Hence both $w_{m+3}$ and $M^{*}$ are less than $w_{m+2}$, we have

$$
\begin{aligned}
w_{m+2} & <w_{m+2} \mu_{m+2}(L \cup M)+R^{*} \mu_{m+2}(R) \\
& <w_{m+2} \mu_{m+1}(L \cup M)+R^{*} \mu_{m+1}(R)=w_{m+1},
\end{aligned}
$$

since $R^{*}>w_{m+2}$ and the weighting of the convex combinations of these two numbers shifts in the second equation towards the larger one.

It is worth noting that the simpler strategy pair, where in period $i$ males accept any female $y>w_{i+1}$ and females accept any male $x>z_{i+1}$, has the same mating pattern and is a weak equilibrium in the sense that if everyone is following this strategy no one can improve his/her utility by a unilateral change. However it fails to take advantage of errors by a high fitness matched partner who accepts, and does not meet the 'only if' part of our explicit equilibrium condition (1). In a sense it is not subgame perfect. On the other hand, this simple strategy pair has the property that individuals do not need to know their own fitness levels, and for this reason may have applications where the assumption of self-knowledge is not warranted. The problem of learning one's own fitness level through observing the pattern of your acceptance/rejection by others is thus not accessible in our model.

\section{Existence of equilibria}

In this section we show how Theorem 1 can be used to reduce the strategy set to a subset of a finite dimensional space, where Brouwer's Fixed Point Theorem can then be applied to establish that some strategy is an equilibrium. For simplicity of presentation we assume in this section that the reserve values $w_{n+1}$ and $z_{n+1}$ are both zero.

According to Theorem 1, any equilibrium strategy pair must be of the following type (setting $\left.a_{i}=w_{i+1}, b_{i}=z_{i+1}\right)$.

Definition: A strategy pair $(f, g)$ is called a stepwise strategy pair for the game $\Gamma_{n}\left(F_{1}, G_{1}\right)$ if it is given in the following form, for some $A=\left(a_{1}, a_{2}, \ldots, a_{n-1}\right)$ and $B=\left(b_{1}, \ldots, b_{n-1}\right)$ with $a_{i}$ and $b_{i}$ both strictly decreasing, and $a_{n}=b_{n}=0$, where for each period $m=1, \ldots, n-1$ we have:

$$
\begin{aligned}
& f_{m}(x)= \begin{cases}b_{k-1}, & \text { if } x \in\left(a_{k}, a_{k-1}\right), k \geq m+2, \\
b_{m}, & \text { if } x>a_{m+1} .\end{cases} \\
& g_{m}(x)= \begin{cases}a_{k-1}, & \text { if } x \in\left(b_{k}, b_{k-1}\right), k \geq m+2, \\
a_{m}, & \text { if } x>b_{m+1} .\end{cases}
\end{aligned}
$$


In this case we will denote the stepwise strategy pair simply by $(A, B)$. A single strategy which is given in this form will be called a stepwise strategy.

The set of all stepwise strategies $A$ will be denoted by $\Delta_{n-1}$. This set is a closed convex subset of $R^{n-1}$. The set of all stepwise strategy pairs will be denoted by $S=\Delta_{n-1} \times \Delta_{n-1}$, and is also closed and convex. For fixed $n, F_{1}, F_{2}$, we define a mapping

$$
V: S \rightarrow S
$$

as follows. Given a stepwise strategy pair $(A, B) \in S$, we use (6) to determine the resulting distributions $F_{i}, G_{i}, i=2, \ldots, n$, and then use (9) to calculate the corresponding values $w_{i}$ and $z_{i}, i=2, \ldots, n$ as

$$
\begin{aligned}
& w_{i}=a_{i} F_{i}\left(a_{i}\right)+\int_{a_{i}}^{1} x d F_{i}(x), \\
& z_{i}=b_{i} G_{i}\left(b_{i}\right)+\int_{b_{i}}^{1} y d G_{i}(y) .
\end{aligned}
$$

The mapping $V$ is then defined by

$$
V\left(\left(a_{1}, \ldots, a_{n-1}\right),\left(b_{1}, \ldots, b_{n-1}\right)\right)=\left(\left(z_{2}, \ldots, z_{n}\right),\left(w_{2}, \ldots, w_{n}\right)\right) .
$$

Any fixed point of $V$ will be an equilibrium strategy pair for $\Gamma_{n}\left(F_{1}, F_{2}\right)$ as illustrated in the proof of Theorem 1 .

To show that $V$ is continuous we will only show the first step, that $w_{2}$ (which depends only on $a_{1}, a_{2}, b_{1}, F_{1}$ and $G_{1}$ ) is a continuous function of $a_{1} a_{2}$ and $b_{1}$. Note that a male $x$ will remain in the population to period 2 definitely if $x \leq a$ and with probability $G(b)$ (that is, if he meets a $y<b$ ) if $x>a$. Consequently the total population of males (also of females) at the beginning of period 2 is given by

$$
T_{2}=F_{1}\left(a_{1}\right)+\left(1-F_{1}\left(a_{1}\right)\right) G_{1}\left(b_{1}\right)=F_{1}\left(a_{1}\right)+G_{1}\left(b_{1}\right)-F_{1}\left(a_{1}\right) G_{1}\left(b_{1}\right),
$$

which is continuous in $a$ and $b$. Furthermore, the normalized male population distribution $F_{2}$ at the beginning of period 2 is given by

$$
F_{2}(x)= \begin{cases}\frac{F_{1}(x)}{T_{2}\left(a_{1}, b_{1}\right)}, & \text { if } x \leq a_{1}, \\ \frac{F_{1}\left(a_{1}\right)\left(1-G_{1}\left(b_{1}\right)\right)+G_{1}\left(b_{1}\right) F_{1}(x)}{T_{2}\left(a_{1}, b_{1}\right)}, & \text { if } x>a_{1} .\end{cases}
$$

Consequently $w_{2}$ is given by

$$
\begin{aligned}
w_{2} & =a_{2} F_{2}\left(a_{2}\right)+\int_{a_{2}}^{1} x d F_{2}(x) \\
& =a_{2} F_{2}\left(a_{2}\right)+\int_{a_{2}}^{a_{1}} x d F_{2}(x)+\int_{a_{1}}^{1} x d F_{2}(x) \\
& =\frac{1}{T_{2}}\left(a_{2} F_{1}\left(a_{2}\right)+\int_{a_{2}}^{a_{1}} x d F_{1}(x)+G_{1}\left(b_{1}\right) \int_{a_{1}}^{1} x d F_{1}(x)\right),
\end{aligned}
$$


which is continuous in $a_{1}, a_{2}$, and $b_{1}$, if $F_{1}$ and $G_{1}$ are continuous. Similar calculations show that all the $w_{i}$ and $z_{i}, i=2, \ldots, n$, are continuous functions of $\left(\left(a_{1}, \ldots, a_{n-1}\right),\left(b_{1}, \ldots, b_{n-1}\right)\right)$.

Since $V$ is continuous and maps the closed convex subset $S$ of $R^{n-1}$ into itself, it has a fixed point by Brouwer's Theorem. This fixed point must be an equilibrium stepwise strategy pair. Thus we have established the following.

Theorem 2 Suppose the initial population distributions $F_{1}$ and $F_{2}$, of males and females, are continuous. Then the cohort mating game $\Gamma_{n}\left(F_{1}, F_{2}\right)$ has an equilibrium strategy pair.

In a similar manner, we can define the symmetric version of the map $V$, as

$$
V^{\text {sym }}: \Delta_{n-1} \rightarrow \Delta_{n-1},
$$

which gives the common sequence of $w_{i}$ 's when both players adopt the same stepwise strategy, obtaining the symmetric form of the previous result

Theorem 3 Every symmetric game $\Gamma(F)$, with $F$ continuous, has a symmetric equilibrium.

\section{Multiple, Mixed, and Asymmetric Equilibria}

In this section we analyze $\Gamma(F)$ for a symmetric discrete distribution $\hat{F}$ having three fitness levels for which there are multiple pure equilibria and also a mixed strategy equilibrium for the two period game. However if the two equilibria are adopted (one each) by the two players, the resulting strategy pair is not an equilibrium. Furthermore we show that no two period symmetric mating game can have an asymmetric equilibrium.

Consider a population with initial distribution $\hat{F}$ equally divided (1/3 each) between fitness levels $0, .1$, and .3. Clearly at equilibrium a 0 will never be accepted in period 1, while a .3 will always be accepted. Consequently the two possible equilibrium strategies in period 1 are 'high' (accepting only .3), or 'middle' (accepting only .1 or .3). We show that both of these strategies are symmetric equilibria for the $n=2$ period game.

First consider the 'high' strategy. In this case the only matings that occur in period 1 are between two .3 individuals. The population in period 2 has $1 / 3$ of $0,1 / 3$ of .1 , and $2 / 9$ of .3 , with a total population of $8 / 9$. The mean fitness of the period 2 population is

$$
\frac{\left(\frac{1}{3}\right) 0+\left(\frac{1}{3}\right) \cdot 1+\left(\frac{2}{9}\right) \cdot 3}{\frac{8}{9}}=.1125 .
$$

Since

$$
.1<.1125<.3 \text {, }
$$


it is consistent (equilibrium behavior) for all individuals to reject a .1 in period 1 (receiving on average .1125 in the next period), but to accept a .3. Thus the strategy of accepting only .3's in period 1 is an equilibrium. (It will remain so even if there is a cost $c<.0125$ of entering the next period.) The inter-couple correlation coefficient at this 'high' equilibrium is $\rho=\frac{33}{112} \doteq .2946$.

Next consider the 'middle' strategy of accepting .1 or .3 in period 1 . In this case any match not involving a 0 in period 1 will result in a mating. The population in period 2 will have a population with mass $1 / 3$ of 0 's, $1 / 9$ of.1's and $1 / 9$ of .3 's, with a total population of $5 / 9$. This is because a .1 or a .3 will have a $2 / 3$ probability of mating in period 1 . The population mean in period 2 will be

$$
\frac{\left(\frac{1}{3}\right) 0+\left(\frac{1}{9}\right) .1+\left(\frac{1}{9}\right) .3}{\frac{5}{9}}=0.08 .
$$

Since

$$
0<.08<.1,
$$

it is consistent to accept a .1 in period 1 , rather than getting an average of .08 in period 2. Hence this is also an equilibrium. The correlation coefficient at this equilibrium is $\frac{32}{105} \doteq .3047$. (This will remain an equilibrium even if there is a cost $c<.08$ of entering the next period, so if $.0125<c<.08$ this would be the only equilibrium.)

It is worth observing that if the cost is .0125 and the equilibrium is the 'high' one with correlation $\rho=.2946$ and the cost is increased slightly, then the new 'middle' equilibrium will have a greater correlation of $\rho=.3047$, so that increasing the entry cost $c$ can theoretically increase the inter-couple correlation. This behavior is the opposite of that found by Johnstone (1997) and in this article in Section 3.

The above atomic distribution $\hat{F}$ concentrated on the three levels $0, .1$, and .3 can be approximated by one with a continuous cumulative distribution (only atom is at left end 0 ), and such a distribution will also have multiple equilibria. To this end, consider the continuous distribution $F$ (shown in Figure 8) which gives probability .32 to fitness 0 , and is uniform on the following intervals with the total probabilities given: .02 on $(0, .098), .32$ on $(.098, .102), .02$ on 
$(.102, .298)$, and .32 on $(.298, .302)$.

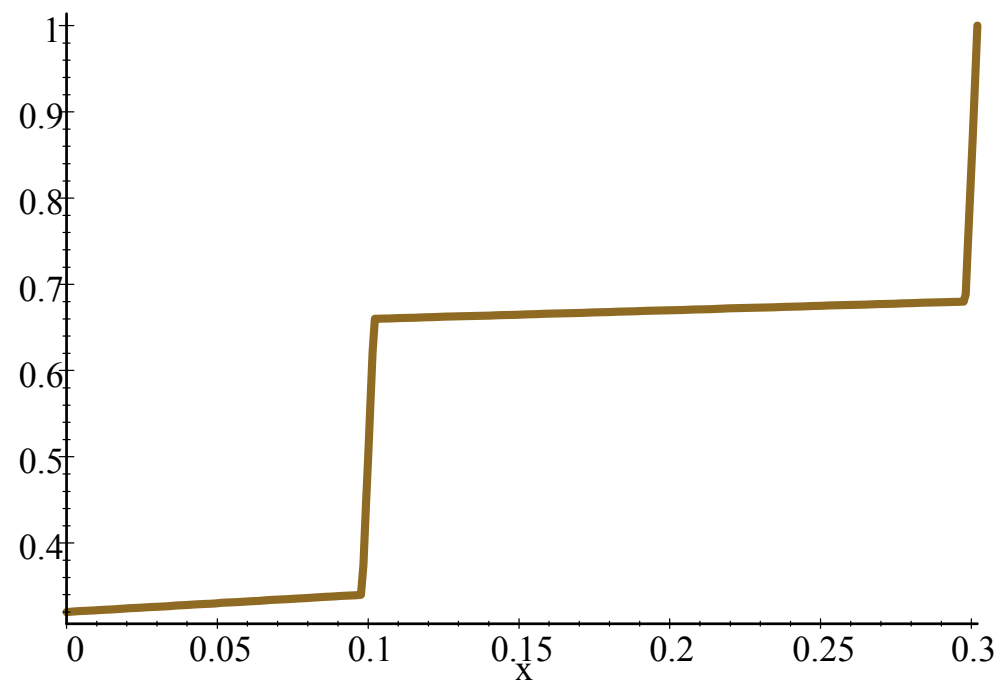

Figure 8: An approximating continuous distribution

In Figure 9 we plot the mean fitness in period 2 as a function of the acceptance level $x$ in period 1 , so that intersections of this curve with the diagonal constitute equilibrium acceptance strategies $x$.

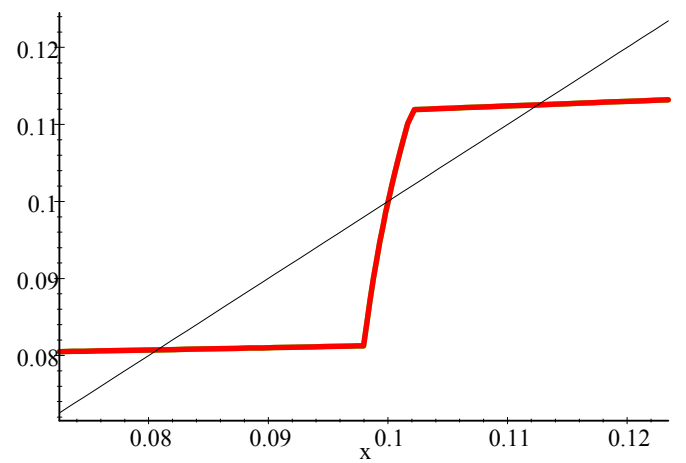

Figure 9: Second period mean fitness

We note that there is at equilibrium near .08 which accepts everyone in the interval $(.098, .102)$ and thus corresponds to the 'low' strategy equilibrium for the atomic distribution, and a similar one near .115 which corresponds to the 
'high' strategy equilibrium. However there is an additional intersection near .1 which does not apparently correspond to either equilibrium of the atomic distribution. Note that it accepts approximately half of the individuals in the interval $(.098, .102)$. We now show that this equilibrium of the continuous distribution corresponds to a mixed strategy for the atomic distribution which accepts a .1 individual with probability $p=1 / 2$.

We return to the atomic distribution and consider now the symmetric mixed strategy $f_{p}$ which in the first period always rejects a 0 , always accepts a .3 , and accepts a .1 with probability $p$. If two .1 players meet in the first period, they randomize independently. The normalized population at the beginning of the second (and last) period has

$$
\frac{3}{8-2 p-p^{2}} \text { level 0's, } \frac{3-p-p^{2}}{8-2 p-p^{2}} \text { level .1's, and } \frac{2-p}{8-2 p-p^{2}} \text { level .3's, }
$$

with a final period mean fitness level of

$$
\lambda(p)=\frac{1}{10} \frac{3-p-p^{2}}{8-2 p-p^{2}}+\frac{3}{10} \frac{2-p}{8-2 p-p^{2}}=\left(\frac{1}{10}\right) \frac{-9+4 p+p^{2}}{-8+2 p+p^{2}} .
$$

The mean fitness $\lambda(p)$ is strictly decreasing, with $\lambda(1 / 2)=.1$. Consequently when the strategy $p=1 / 2$ is adopted, a player is indifferent between accepting a player with fitness .1 in period 1 and going into the next period and getting on average .1. Thus the mixed strategy with $p=1 / 2$ is an equilibrium strategy.

\subsection{Relative fitness}

There is another method of modeling the distribution of fitness levels in the population which is more complicated but incorporates atoms and illustrates mixed strategies. For simplicity we will only consider the symmetric problem with a common initial distribution $F$ of fitness. We let $r, 0 \leq r \leq 1$ denote the relative fitness of an individual. That is, an individual has relative fitness $r$ if the fraction of the population with lower fitness is $r$. The variable $r$ is always uniformly distributed on $[0,1]$. An individual with absolute fitness $x$ has relative fitness $r=F(x)$. This is fine as long as $F$ has no atoms. To see how we deal with atoms, recall the atomic distribution $\hat{F}$ with three equal mass atoms at absolute fitness $0, .1$, and .3. We will assume that say the individuals with fitness .1 have their relative fitness levels $r$ uniformly distributed over the interval $[1 / 3,2 / 3]$. We could interpret this as their each having a second (possibly unobservable) fitness indicator which distinguishes among them. This second indicator however contributes nothing to absolute fitness, or to the utility their mate gets. We define $x=\phi(r)$ to be the absolute fitness level of an individual of relative fitness level $r$. In order to have the graph of absolute fitness be a closed set, we define the absolute fitness interval $\Psi(r)$ to be the closed interval from the left limit to the right limit of $\phi$ at $r$. Thus if $\phi$ is continuous at $r$, then $\Psi(r)$ is simply the single point $\phi(r)$. The function $\Psi(r)$ corresponding to the distribution $\hat{F}$ is drawn in Figure 10. (Basically, it is just 
the function $\phi(r)$, with vertical lines connecting the discontinuities resulting from the 'empty' fitness intervals $(0, .1)$ and $(.1, .3)$ which contain no individuals. Note that $\Psi(2 / 3)=[.1, .3]$, which is the interval cited in (20) which contains the mean fitness .1125. Similarly $\Psi(1 / 3)=[0, .1]$ which contains the mean fitness level .08.

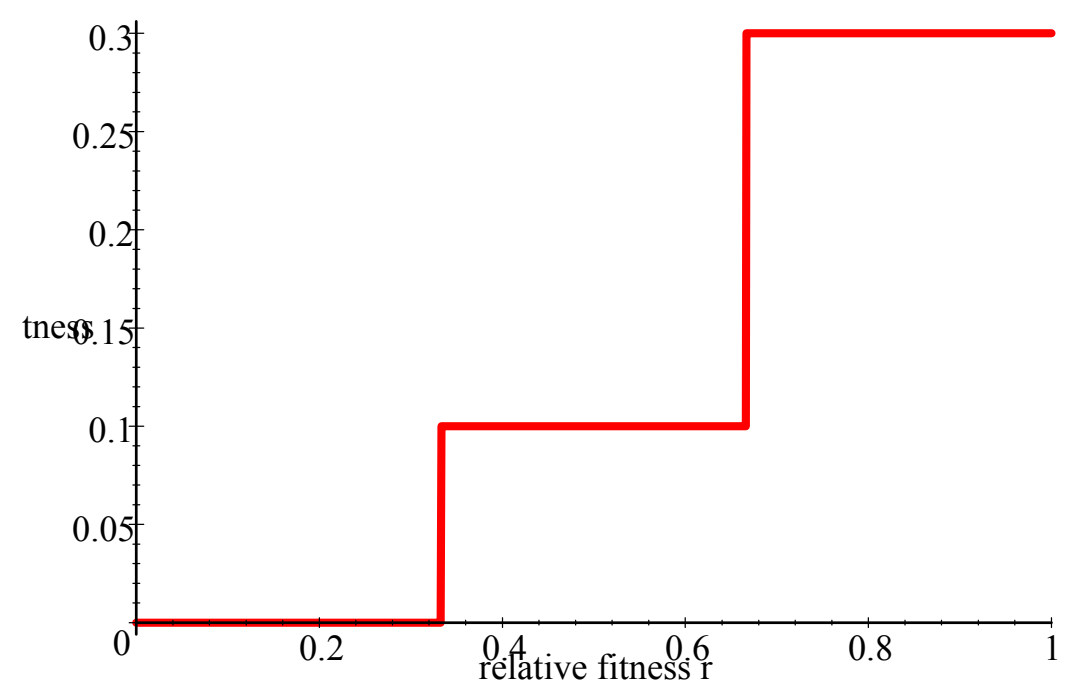

Figure 10: Absolute as function of relative fitness

We now calculate the mean second period absolute fitness as a function of the first period relative acceptance level $r$. In the case that $\Psi(r)$ is a single fitness level $x$, the strategy with relative acceptance level $r$ simply accepts all absolute fitness levels above $x$, or equivalently, all relative fitness levels above $r$. However in a case such as $r \in(1 / 3,2 / 3)$, which corresponds to the atom at .1 , we need another more refined version. We interpret this as a mixed strategy which accepts all fitness levels above .1 (in this case .3) and accepts those with .1 with probability proportional to the fraction of the interval $[1 / 3,2 / 3]$ lying above $r$. The probability $p$ corresponding to $r$ is given by $p(r)=(2 / 3-r) /(2 / 3-1 / 3)=$ $2-3 r$. Making this substitution in the formula given in (23) for $\lambda(p)$ gives the mean absolute fitness $m(r)$ in period 2 as a function $m(r)$ of relative fitness $r$, for $1 / 3 \leq r \leq 2 / 3$, which we plot along with $\Psi(r)$ in Figure 11 . The function $m(r)$ is a continuous function which intersects the graph of $\Psi$ in the three points where $m(r) \in . \Psi(r)$. The intersections where $r$ is $1 / 3$ and $2 / 3$ correspond to the 'middle' and 'high' equilibria already observed in inequalities (22) and (20), which in our new notation would be written as

$$
\begin{aligned}
.1125 & =m(2 / 3) \in \Psi(2 / 3)=[.1, .3], \text { and } \\
.08 & =m(1 / 3) \in \Psi(1 / 3)=[0, .1] .
\end{aligned}
$$


The remaining intersection at $r=1 / 2$ corresponds to the mixed equilibrium for $\hat{F}$ which accepts $p=1 / 2=2-3(1 / 2)$ of the individuals with absolute fitness .1 and all of those with .3 .

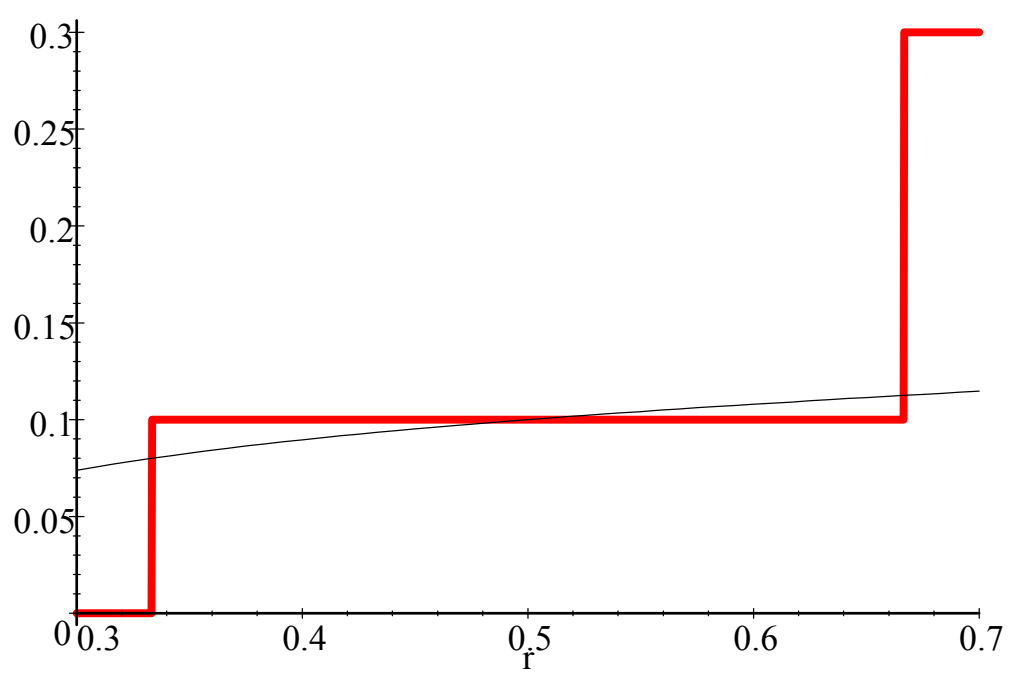

Figure 11: Three intersections $m(r) \in \Psi(r)$ are equilibria

The advantage of this type of modelling (with relative fitness) is that mixed and pure equilibria are found by a common method. The fact that there is at least one intersection $m(r) \in \Psi(r)$ is intuitive for the two period model (allowing non-atomic distributions), and we believe that a more general existence result can be obtained in this way for the $n$ period model via Kakutani's Fixed Point Theorem.

\subsection{Asymmetric equilibria}

We have already seen in Section 3 an example of an asymmetric equilibrium occurring when the problem is asymmetric (entry cost for females only). On the other hand we know by Theorem 4 that for a symmetric game $\left(F_{1}=G_{1}\right)$ there is always a symmetric equilibrium. Now that we also know that there can be multiple equilibria, even for $n=2$, it is worth asking whether there can be another equilibrium for a symmetric game that is not symmetric (with $f \neq g$ ). While we cannot answer this question fully, we can demonstrate by a straightforward analysis that it is not possible for $n=2$.

Theorem 4 If $(f, g)$ is an equilibrium for the two period symmetric game $\Gamma_{2}(F)=$ $\Gamma_{2}(F, F)$, then $f=g$. 
Proof. Suppose that $f \neq g$. Then without loss of generality we may assume that $f$ and $g$ have minimum first period acceptance levels of $a$ and $b$, respectively, with

$$
a>b \text { and } 1>F(a)>F(b) .
$$

(Note that the second condition is implied by the equivalence of $f$ and $g$, since otherwise the two strategies differ only on accepting fitness levels which do not exist in the population.)

The two numbers $a$ and $b$ determine three intervals $[0, b],(b, a]$, and $(a, 1]$, for which the mean fitness increases from left to right. In particular, we have that ( $\mathrm{M}$ for middle, $\mathrm{R}$ for right)

$$
M \equiv \frac{\int_{b}^{a} x d F(x)}{(F(a)-F(b))}<a<R \equiv \frac{\int_{a}^{1} x d F(x)}{1-F(a)} .
$$

Since matings will occur in period 1 if and only if $x>b$ and $y>a$, the total population at the beginning of period 2 for both males and females is given by

$$
F(a)+F(b)-F(a) F(b),
$$

which we will denote simply by $T$. The normalized population of males at the beginning of period 2 is given by

$$
G_{2}(x)=\frac{1}{T} \begin{cases}F(x), & \text { if } x \leq b, \\ F(a) F(x), & \text { if } x>b .\end{cases}
$$

Consequently the mean fitness of period 2 males is given by

$$
w_{2}=\frac{1}{T}\left[\int_{0}^{b} x d F(x)+F(a) \int_{b}^{1} x d F(x)\right] .
$$

Similarly, the mean fitness of period 2 females is given by

$$
z_{2}=\frac{1}{T}\left[\int_{0}^{a} y d F(y)+F(b) \int_{a}^{1} y d F(y)\right] .
$$

We calculate

$$
\begin{aligned}
T\left(z_{2}-w_{2}\right) & =(1-F(a)) \int_{b}^{a} x d F(x)-(F(a)-F(b)) \int_{a}^{1} x d F(x), \\
\frac{T\left(z_{2}-w_{2}\right)}{(1-F(a))(F(a)-F(b))} & =\frac{\int_{b}^{a} x d F(x)}{(F(a)-F(b))}-\frac{\int_{a}^{1} x d F(x)}{1-F(a)} \\
& =M-R<0 .
\end{aligned}
$$

Consequently we have

$$
z_{2}<w_{2}
$$


However if the pair $(a, b)$ is an equilibrium we have

$$
a=z_{2}, b=w_{2} \text {, and hence } b>a,
$$

which is contrary to our assumption.

It is possible that this type of monotonicity argument can be extended to any number of periods.

\subsection{No universal acceptance}

The formulae developed in the previous section for second period mean fitness can now be used, in the symmetric form, to show that the equilibrium strategy never features universal acceptance $(a=0)$. We need to show that the limit of second period mean fitness, as $a$ goes to 0 , always is positive. Hence individuals of sufficiently low but positive fitness will be rejected at equilibrium. In fact we will show that when $F$ has no atoms and is continuously differentiable this limit is exactly half the initial mean fitness. (We have already seen this in Figure 1 , where the uniform distribution has mean $1 / 2$ and the mean second period fitness level goes to $1 / 4$ at 0 .) Note that the symmetric $(a=b)$ form of (25) gives second period mean fitness $w(a)$ as a function of first period acceptance level $a$,

$$
w(a)=\frac{\int_{0}^{a} x d F(x)+F(a) \int_{a}^{1} x d F(x)}{2 F(a)-F(a)^{2}}
$$

As both numerator and denominator go to zero as $a$ goes to zero, we use l'Hôpital's rule to calculate

$$
\begin{aligned}
\lim _{a \rightarrow 0} w(a) & =\lim _{a \rightarrow 0} \frac{a F^{\prime}(a)+F^{\prime}(a) \int_{a}^{1} x d F(x)}{2 F^{\prime}(a)-2 F(a) F^{\prime}(a)} \\
& =\lim _{a \rightarrow 0} \frac{a+\int_{a}^{1} x d F(x)}{2(1-F(a))}=\frac{\int_{a}^{1} x d F(x)}{2(1-F(a))}, \text { or } \\
& =\frac{1}{2} \int_{0}^{1} x d F(x) \text { (half mean initial fitness), }
\end{aligned}
$$

when there is no atom at 0 . Since in any case this is positive, we have shown that.

Theorem 5 If the initial fitness distribution $F$ is continuously differentiable, then the strategy of universal acceptance in period 1 is not an equilibrium for the game $\Gamma_{2}(F)$.

\section{Learning Behavior}

Up to now we have been assuming that the initial fitness distributions $F_{1}$ and $F_{2}$ are common knowledge. This means that they are known to the players, 
all the players know that they are known to the other players, that they are known to be known, and so on. In this section we assume, on the contrary, that Nature chooses the initial distribution from a family of distributions, according to known probabilities. Each player may update this prior distribution over initial distributions according to the fitness levels of the players he is matched with and also according to his own fitness level. We will not develop a general theory for this type of mating problem, but instead we consider in depth a simple example which exhibits some interesting phenomena. An equilibrium strategy in this context is one in which a player accepts only those matches with fitness level above his expected utility if he enters the next period unmated, where the expectation is taken with respect to his posterior probability over the possible initial distributions, based on the players he has encountered as matches.

Consider a symmetric problem with $n=2$ periods, and a reserve value of zero. The only strategic variable in this context is whom to accept as a mate in period 1. Suppose that there are only three fitness levels: 0, a medium level denoted $M$, and a high level denoted $H$, with $0<M<H$. Nature chooses the initial distribution over these three levels equiprobably according to distribution I:( $(1 / 4,1 / 2,1 / 4)$ (that is, 0 with probability $1 / 4, M$ with probability $1 / 2$, and $H$ with probability $1 / 4)$ or distribution II: $(1 / 4,1 / 4,1 / 2)$. Distribution I has more $M$ 's while II has more $H$ 's.

The prior probabilities of I and II are $1 / 2$ and $1 / 2$, but the posterior probabilities for each of a matcher pair $(x, y)$ (order is irrelevant) with $x, y \in\{0, M, H\}$ can be calculated as follows. Only the first three will matter, since a 0 will never be accepted.

$$
\begin{aligned}
p(I \backslash(H, H)) & =1 / 5, P(I I \backslash(H, H))=4 / 5, \\
p(I \backslash(M, M)) & =4 / 5, P(I I \backslash(M, M))=1 / 5, \\
p(I \backslash(M, H)) & =1 / 2, P(I I \backslash(M, H))=1 / 2, \\
p(I \backslash(0, M)) & =2 / 3, P(I I \backslash(0, M))=1 / 3, \\
p(I \backslash(0, H)) & =1 / 3, P(I I \backslash(0, H))=2 / 3 .
\end{aligned}
$$

A strategy must indicate what minimum level a player will accept in period 1 , given the levels of the player and his match, since these two numbers will determine the expected value of the mean fitness in period 2 . The only three unordered pairs of fitness levels that are relevant are $(H, H),(M, M)$, and $(M, H)$. A strategy $[x, y, z]$ denotes the minimum acceptable mate in each of these cases, respectively. For example, the strategy $[H, M, H]$ says to accept only $H$ if you and your partner are both $H$ (so accept this partner), accept $M$ or $H$ if you and your match are both $M$ (which results in a mating), accept only $H$ if you are $H$ and your partner is $M$ or the other way around (leads to no mating). Thus with this strategy (which we shall show is an equilibrium for certain values of $M$ and $H$ ) the only pairs which mate in period 1 are both $M$ or both $H$.

We now analyze three strategies, $[H, H, H],[M, M, M]$, and $[H, M, H]$, and show that each is an equilibrium for some values of $H / M$, as shown in Figure 
12.

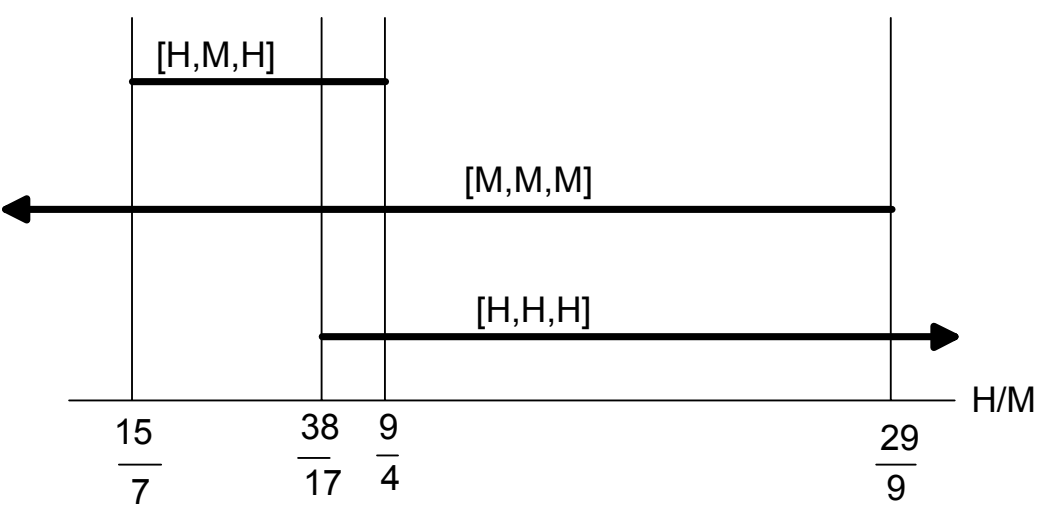

Figure 12: Learning Equilibria for different $\mathrm{H} / \mathrm{M}$

We show that $[H, H, H]$ is an equilibrium for $H / M \geq 38 / 17,[M, M, M]$ is an equilibrium for $H / M \leq 29 / 9$, and $[H, M, H]$ is an equilibrium for $H / M$ between $15 / 7$ and $38 / 17$. Clearly multiple equilibria occur in some cases.

First consider the strategy $[H, H, H]$. If the actual initial distribution is I then we calculate the distribution at the beginning of period 2 as follows. With $[H, H, H]$, the only pairs that will mate are $(H, H)$. Thus the populations of 0 and $M$ will remain $1 / 4$ and $1 / 2$, respectively. An $H$ will be mated if and only if it is matched with another $H$, which will occur according to the initial probability of being an $H$, that is, $1 / 4$. So an $H$ will remain unmated with probability $3 / 4$, and hence the population of $H$ in period 2 will be $(1 / 4)(3 / 4)=3 / 16$. Hence the total population in period 2 is $15 / 16$, and the normalized distribution has $4 / 15$ zero, 8/15 for $M$, and $3 / 15$ for $H$. Consequently the population mean, given initial distribution I and strategy $[H, H, H]$, is given by

$$
\mu_{I}[H, H, H]=\frac{8 M+3 H}{15} .
$$

In a similar manner we calculate the population mean given initial distribution II as

$$
\mu_{I I}[H, H, H]=\frac{5 M+5 H}{15} .
$$

Now we use the posterior probabilities determined above in (26) to calculate the expected population means in period 2, given the fitness levels of a player and his match in period 1 , as

$$
\begin{aligned}
V_{(H, H)}[H, H, H] & =\frac{1}{5}\left(\frac{8 M+3 H}{15}\right)+\frac{4}{5}\left(\frac{5 M+5 H}{15}\right)=\frac{28 M+23 H}{75}, \\
V_{(M, M)}[H, H, H] & =\frac{4}{5}\left(\frac{8 M+3 H}{15}\right)+\frac{1}{5}\left(\frac{5 M+5 H}{15}\right)=\frac{37 M+17 H}{75}, \\
V_{(M, H)}[H, H, H] & =\frac{1}{2}\left(\frac{8 M+3 H}{15}\right)+\frac{1}{2}\left(\frac{5 M+5 H}{15}\right)=\frac{13 M+8 H}{30} .
\end{aligned}
$$


Since the strategy $[H, H, H]$ always accepts only $H$, all of these values must exceed $M$ (otherwise it would be optimal to accept $M$ ). The condition that all three of these expressions exceed $M$ is equivalent to the single inequality

$$
H \geq \frac{38}{17} M
$$

In a similar manner, we find that the strategy $[M, M, M]$ is a Bayesian equilibrium if the three values $V_{(H, H)}[M, M, M], V_{(M, M)}[M, M, M]$, and $V_{(M, H)}[M, M, M]$ are all below $M$ (otherwise $M$ should not be accepted), which is equivalent to the single numerical condition on $M$ and $H$ given by

$$
H \leq \frac{29}{9} M
$$

Finally, we consider the strategy $[H, M, H]$. This will be an equilibrium for values of $M$ and $H$ satisfying the three inequalities

$$
M \leq V_{(H, H)}[H, M, H], M \geq V_{(M, M)}[H, M, H], M \leq V_{(M, H)}[H, M, H] .
$$

The first inequality says that individuals in a matched pair $(H, H)$ will not accept less than $H$ (the first entry in the strategy triple) because their posteriors on initial distributions $I$ and $I I$ lead them to believe the mean of the period 2 population is more than $M$. (So they will accept each other.) The next inequality says that two $M$ 's who meet in period 1 should accept $M$ (the middle entry in the strategy) or above (in particular, each other), because their posteriors imply that they will get less than $M$ if they go unmated into the next period. The final inequality says that if an $M$ and an $H$ meet in period 1 they should each accept only $H$ (final entry in the strategy) because they will get on average more than $M$ in the next round. The $M$ will accept the $H$ but the $H$ will not accept the $M$, so they will not be mated.

Using the top three posterior probabilities calculated in (26), we determine the values $V_{(x, y)}$ giving the expected value of the mean of the period 2 population, calculated according to the posteriors of two individuals $x$ and $y$ who meet in period 1 .

$$
\begin{aligned}
V_{(H, H)} & =\frac{1}{5}\left(\frac{4 M+3 H}{11}\right)+\frac{4}{5}\left(\frac{3 M+4 H}{11}\right)=\frac{16 M+19 H}{55} \\
V_{(M, M)} & =\frac{4}{5}\left(\frac{4 M+3 H}{11}\right)+\frac{1}{5}\left(\frac{3 M+4 H}{11}\right)=\frac{19 M+16 H}{55} \\
V_{(H, H)} & =\frac{1}{2}\left(\frac{4 M+3 H}{11}\right)+\frac{1}{2}\left(\frac{3 M+4 H}{11}\right)=\frac{7 M+7 H}{22} .
\end{aligned}
$$

The equilibrium inequalities (28) for strategy $[H, M, H]$ now translate into the consistent inequalities

$$
\frac{15 M}{7} \leq H \leq \frac{9 M}{4}
$$


For values of $M$ and $H$ satisfying (29), the strategy $[H, M, H]$ is an equilibrium. In this equilibrium the only matings in the first period are between two $M$ 's or between two H's. This pattern of 'like mating only with like' is reminiscent of that in the steady state model of MacNamara and Collins (1990).

\section{Computed Equilibria for $\Gamma(n)$}

In this section we give the results of computations of the equilibria for the symmetric mating game with the uniform distribution of fitness, $\Gamma(n)$. We have already determined the unique equilibria for $n=2$ and 3 analytically. The remaining results given here were obtained by iterating the mapping $V^{\text {sym }}$ defined in (18), whose fixed points constitute the equilibria of $\Gamma(n)$. A clear pattern emerges, as shown in Figure 13. Above each integer point $n$, there are plotted the $n-1$ acceptance points $a_{1}>a_{2}>\cdots>a_{n-1}$ in the symmetric equilibrium for that value of $n$. This technique does not establish uniqueness.

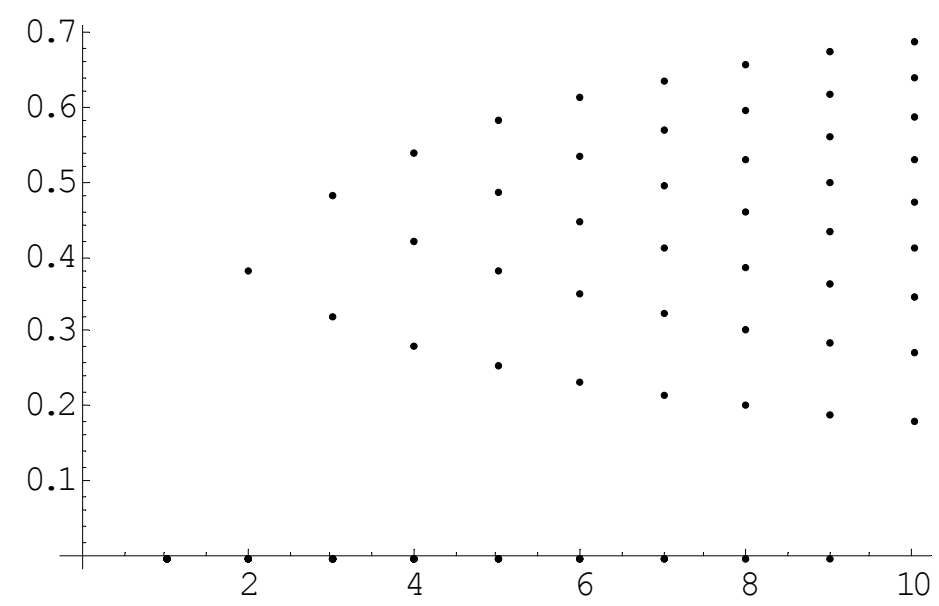

Figure 13: Equilibrium strategies, $\Gamma(n), n \leq 10$

It is interesting to note the pattern of matings obtained by the end of the last period. In particular, these mating patterns may be compared to those obtained in Alpern and Reyniers (1999) and Kalick and Hamilton (1986) for similarity (homotypic) preferences, when dealing with empirical results. A comparison between the mating patterns for the two preference types is planned for a future article. In the Figures 14 and 15 we see the final mating pattern achieved at the end of period $n=3,4$. Lighter areas indicate higher density of matings, but even the darkest areas in all these figures have some matings. As in the MacNamaraCollins steady state model, matings are more likely in squares located on the diagonal (like with like), but unlike their model we also obtain off diagonal 
matings.

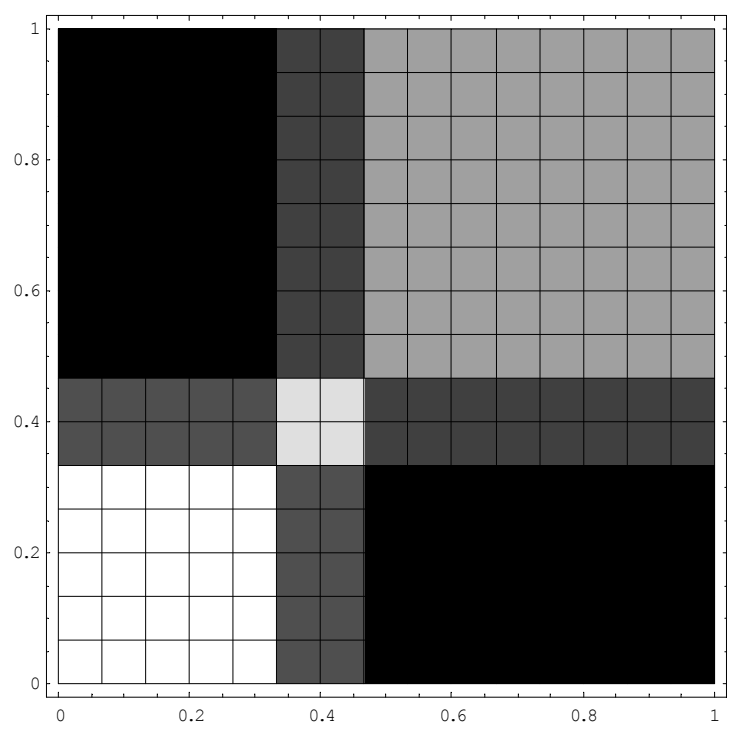

Figure 14: Final mating patterns in $\Gamma(3)$

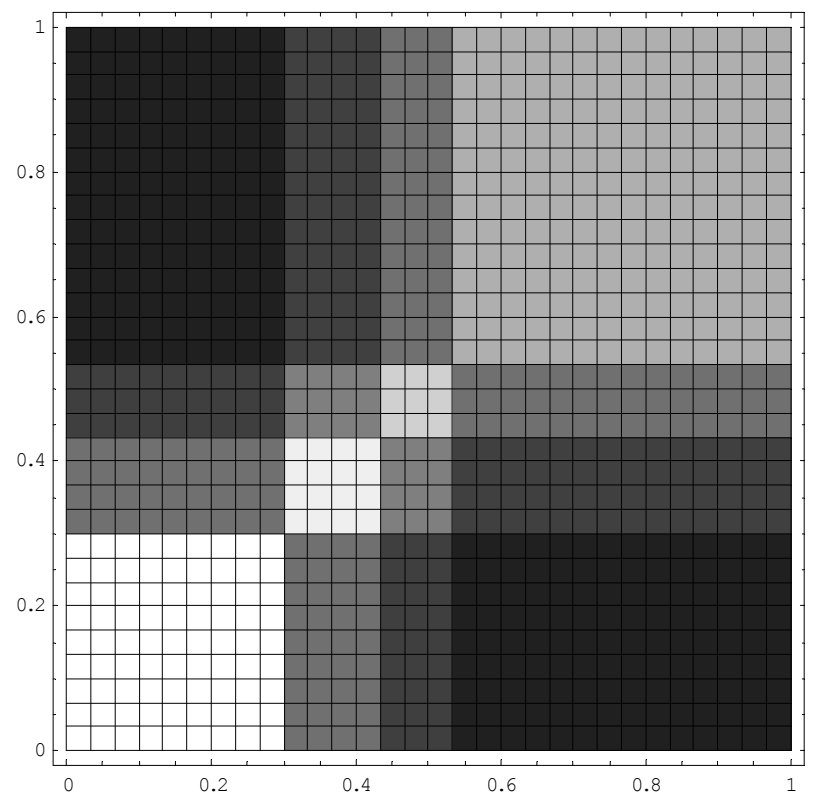

Figure 15: Final mating patterns in $\Gamma(4)$.

We can also look at the mating pattern over time. In the next three figures, we show the patterns of matings in each period 1,2,3, for the game $\Gamma(3)$. Again, 
lighter areas have a higher density of matings, and in periods 1 and 2 the darkest area has no matings (consistent with Theorem 1). In the final period, matings are possible for every pair type, so the density has a positive minimum.

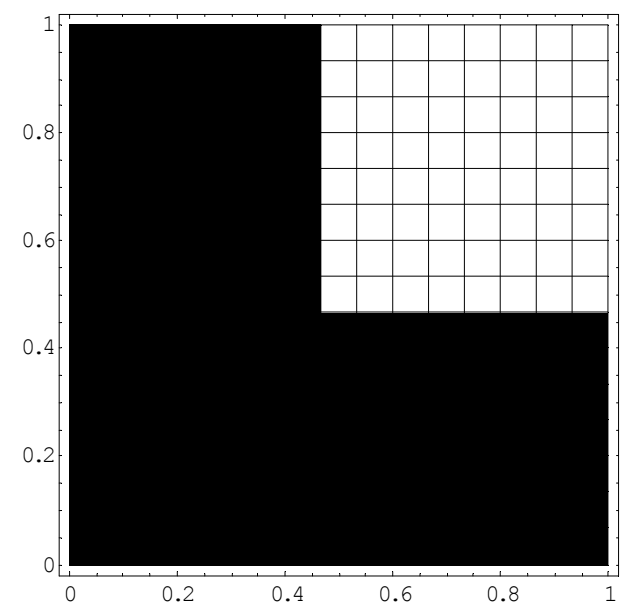

Figure 16: First period matings.

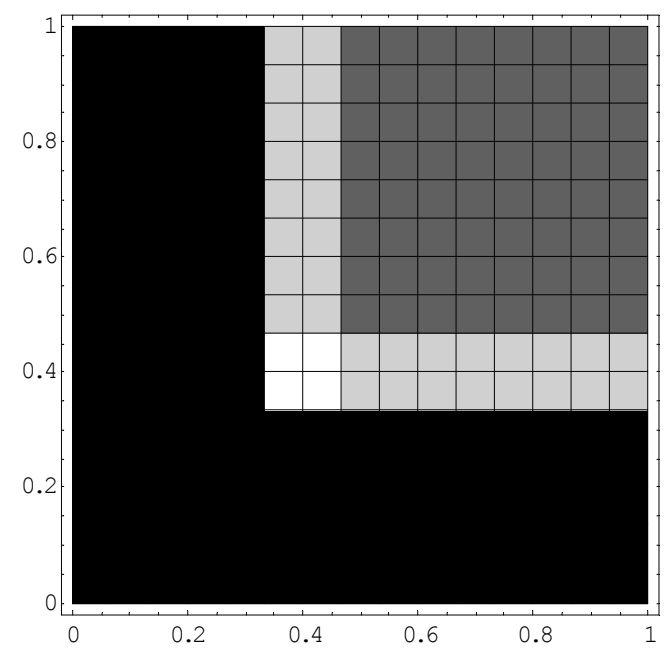

Figure 17: Second period matings. 


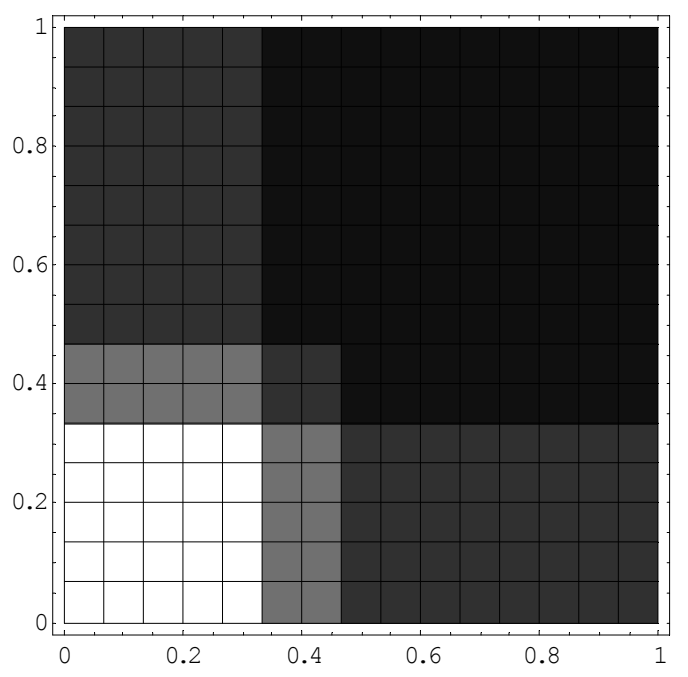

Figure 18: Third period matings.

\section{Conclusions}

The cohort mating game $\Gamma_{n}\left(F_{1}, G_{1}\right)$, which we analyze in this paper, provides a setting for questions about equilibrium mating behavior in a context where meeting is by chance and subsequent mating is by mutual choice. Our basic results (Theorems 1 and 2) on stepwise equilibria, where male and female acceptance levels go down in discrete steps in each period, confirm for arbitrary fitness distributions the results obtained computationally by Johnstone (1997) in a similar model for specific distributions. Our model extends his by allowing consideration of sex differences in fitness distribution or delay costs, although we do not go nearly so far in this direction as in the infinite horizon model of Johnstone et al (1996).

From another point of view, our results complement those in our previous paper (Alpern and Reyniers, 1999), which viewed the same game theoretic extension of the Kalick and Hamilton (1986) dating simulation model for the alternative assumption of homotypic preferences. With the equilibria corresponding to both assumptions now understood and computable, it may be possible to see which better fits empirical mating patterns in various contexts. In this sense, our two papers, taken together, make a contribution to the discussion of the "matching hypothesis" regarding attractiveness levels that has been taking place in the social psychology literature.

With reference to the biological literature, the present paper shows that it may be necessary to consider certain questions on mating equilibria that have not previously been considered. In particular, multiple equilibria and mixed strategy equilibria. In the case where multiple equilibria exist, it would be interesting to see if different populations of the same species exhibit differing equilibria, and which are the determining external factors. We have also begun, 
in a limited way, to examine the role of learning behavior in mating games.

Finally, we mention a number of variations and complications of our model that will have to be considered to make our results applicable to a wider variety of behavior. First, our assumption that matching is random must be relaxed. It is known, for example (Cronin (1991), that for some species the arrival time at the breeding location is size-related. This alone would account for some of the assortative mating. While this can to some extent be modeled by relaxing our assumption that all individuals arrive simultaneously at the beginning of period one, we may also have to assume that the probability of say a male $x$ of meeting a female $y$ in period $m$ is given by some given measure preserving bijection between remaining males and remaining females. We are aware that our model may well assume the possibility of strategies that are more complex than animals can carry out. We may have to restrict the strategy space to much simpler heuristic behavior, such as that of the Mottled Sculpin (Cottus bairdi), where the female will mate with a male only if it is larger than the last male encountered (Cronin, 1991). Such bounded rationality models are common in economic models. It may also prove necessary to model situations in which an individual can return to mate with a previously rejected individual. Two other extensions of the model were suggested by a referee: One is imperfect assessment of partner fitness and the other is varying the sex ratio (the later is of course a special case of using different initial fitness distributions for the sexes).

\section{Bibliography}

Alpern, S., and Reyniers, D. J. (1999). Strategic mating with homotypic preferences. J. Theo. Biol. 198, 71-88.

Bergstrom, C. T., and Real, L. A. (2000). Towards a theory of mutual mate choice: Lessons from two-sided matching. Evolutionary Ecology Research 2, 493-508.

Bloch, F., and Ryder, H. (2000). Two-sided search, marriages, and matchmakers. International Economic Review 41, 93-114.

Burdett, K., and Coles, M. (1997), Marriage and class. Quarterly Journal of Economics 112, 141-168.

Burdett, K., and Coles, M. (1999). Long term partnership formation: employment and marriage. Economic Journal, 307-335.

Cronin, H. (1991). The ant and the peacock. Cambridge University Press.

Crowley, P.H., Travers, S. E., Linton, M. C., Cohn, S. L., Sih, A., Sargent, R. C. (1991). Mate density, predation risk and the seasonal sequence of mate choices - a dynamic game. Am Nat 137, 567-596.

Eeckhout, J. (2000). Bilateral search and vertical heterogeneity. International Economic Review 40, 869-888.

Johnstone, R. A. (1997). The tactics of mutual mate choice and competitive search. Behav. Ecol. Sociobiol. 40, 51-59. 
Johnstone, R. A., Reynolds, J. D., and Deutsch, J. C. (1996). Mutual mate choice and sex differences in choosiness. Evolution 50, 1382-1391.

Kalick, S. Michael, and Hamilton, Thomas E. (1986) The matching hypothesis reexamined. Journal of Personality and Social Psychology 51, 673-682.

McNamara, J. M., and Collins, E. J. (1990) The job search problem as an employer-candidate game. Journal of Applied Probability 28, 815-827.

Parker, G. A., (1983) Mate quality and mating decisions. In Mate Choice, ed. P. Bateson, Cambridge University Press, 141-164.

Pennebaker, J. W., Dyer, M. A., Caulkins, R. S., Litowitz, D. L., Ackreman, P. L., Anderson, D. B. and McGraw, K. M. (1979) Don't the girls get prettier at closing time: A country and western application to psychology. Personality and Social Psychology Bulletin 5, 122-125.

Real, L. (1990). Search theory and mate choice. I. Models of single sex discrimination. Am. Nat. 136, 376-404.

Shimer, R., and Smith, L. (2000). Assortative Matching and Search. Econometrica 68, no. 2, 342-370.

Zohar, Ada, and Guttman, Ruth (1989) Mate preference is not mate selection. Behavioral and Brain Sciences 12, 38-39. 